\title{
NIR - Remote Sensing and Artificial Neural Networks for Rapid Identification of Post Consumer Plastics
}

Th. Huth-Fehrea, R. Feldhoffa, Th. Kantimmª, L. Quicka , F. Winter ${ }^{\mathrm{a}}$, K. Cammann ${ }^{\mathrm{a}}$, W. van den Broek ${ }^{b}, D$. Wienke ${ }^{b}$ W. Melssen ${ }^{b}$ and L. Buydens ${ }^{b}$

anstitute for Chemical and Biochemical Sensor Research, Wilhelm-Klemm-Straße 8, D-48149 Münster, Germany

${ }^{b}$ Catholic University Nijmegen, Laboratory for Analytical Chemistry, Toernooiveld 1, 6525 ED Nijmegen, The Netherlands

An imaging spectrometer with a 256 element InGaAs diode array was combined with a high throughput optical arrangement for recording high quality NIR spectra $(824 \mathrm{~nm}$ to $1700 \mathrm{~nm})$ of plastics from a distance of $25 \mathrm{~cm}$ within 6.3 milliseconds. The considered spectral region was assessed to be suitable for plastic identification.

\section{INTRODUCTION}

The recycling of plastics plays an increasingly important role in today's society and large efforts are undertaken to find new ways to reuse post-consumer plastics. The direct reuse of the plastic (material recycling) is a very interesting alternative to incineration (energy recycling) or cracking (back-to-feedstock recycling). Unfortunately direct processing of plastic waste to mixed resins leads to materials of only poor quality (e.g. low mechanical stability). Because of this, several ways of identifying the different components (in combination with mechanical sorting) are in general discussion: Mass spectroscopy, X-ray fluorescence, plasma spectroscopy and vibrational spectroscopy (NIR-, MIR- or Raman-spectroscopy) have been applied by different groups [1]. In this paper we concentrate on a new approach using NIR reflectance and transmittance spectroscopy.

The applicability of NIR spectroscopy to plastic identification has been studied by several groups [2-5]. The main advantage of this technique is simple instrumentation and the fact that spectra can be recorded fast and easily without sample preparation. Furthermore, with one exception the spectra beyond $1000 \mathrm{~nm}$ are nearly independent of the colour of the resin. Only carbon black is a strong absorber in the NIR region, which results in featureless spectra [3].

Until now, all existing NIR identification techniques [2-5] concentrate on the first overtones of the CH-stretching vibrations (between 1650 and $1800 \mathrm{~nm}$ ) [6,7]. These bands produce strong spectral features, allowing of high signal-to-noise ratios (SNR). Spectral ranges from 1000 to $1800 \mathrm{~nm}[2,3]$ or from 1000 to $2500 \mathrm{~nm}$ [5] have been accessed with $\mathrm{Ge}$ or $\mathrm{PbSe}$ detectors respectively. In this work we demonstrate that already the spectral region from 824 to $1700 \mathrm{~nm}$, where only the weak second and third overtones of the stretching vibrations are present, allows of the separation of the 5 major types of plastic. As a consequence of this 0022-2860/95/\$09.50 C 1995 Elsevier Science B.V. All rights reserved SSDI 0022-2860(95)08609-9 
result, $\operatorname{In}_{0.53} \mathrm{Ga}_{0.47} \mathrm{As}$ with a spectral range from 800 to $1700 \mathrm{~nm}$ and sensitivity reaching $D^{*} \sim 10^{13} \mathrm{~cm} \mathrm{~Hz}^{1 / 2} / \mathrm{W}$ at room temperature [8] can be used as detector material.

\section{EXPERIMENTAL}

The aim of the described experiment was to test if a suited spectrometer concept can combine the industrial requirements of robustness and speed with the need of recording high quality spectra from plastics without direct contact between sample and coupling optics. The applicability of multichannel spectroscopy for this task was investigated. In order to chose the optimal detector material, a detailed preliminary study covering the complete NIR spectral region from 1000 to $2500 \mathrm{~nm}$ was carried out [9]. In particular the separation power of different wavelengths was investigated. The most important result was that high SNR spectra from 824 to $1700 \mathrm{~nm}$ can provide information sufficient for plastic identification.

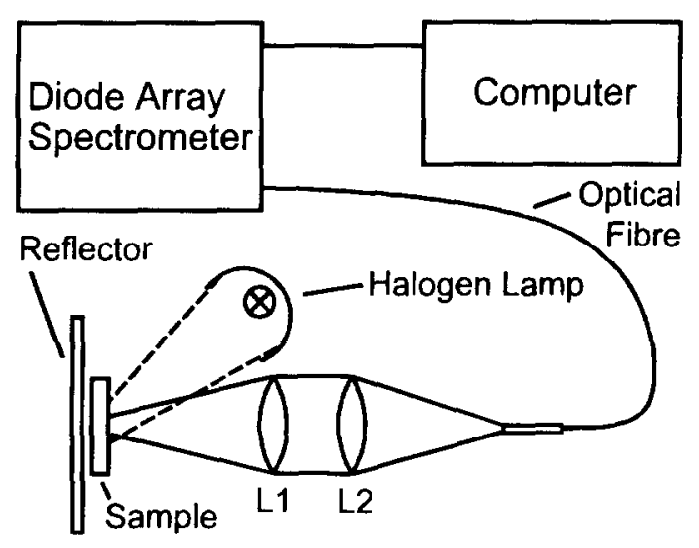

Figure 1. Spectrometer with coupling optics, transflectance arrangement and illumination source

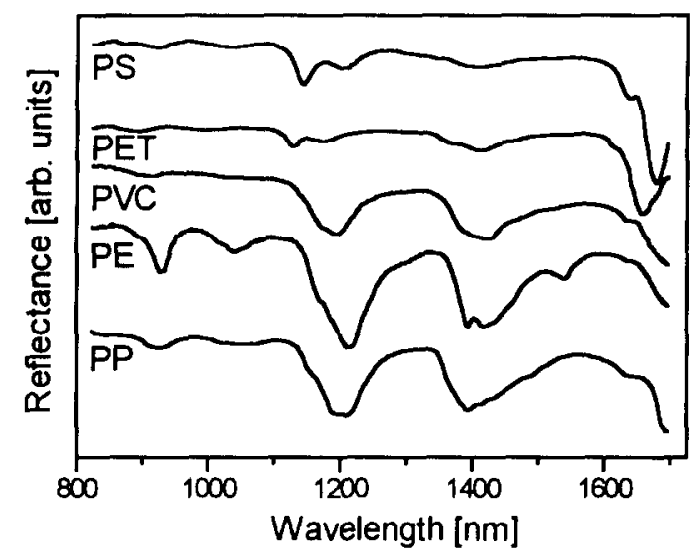

Figure 2. Typical spectra of the 5 major types of plastic contained in household garbage (with different off-sets)

The final experimental set-up is shown in figure 1. The experiment was carried out with an X-DAP spectrometer (Polytec, Germany). This spectrometer uses a $190 \mathrm{~mm} \mathrm{f} / 2.8$ imaging concave grating with 65 grooves/mm (Jobin Yvon). The spectral region from 824 to $1700 \mathrm{~nm}$ is projected onto 232 pixels of a 256-element InGaAs-array (EPITAXX ETX 100MLA 256). With an entrance slit width of $100 \mu \mathrm{m}$ the instrument reaches a resolution of $10 \mathrm{~nm}$. Control and read-out of the room-temperature operated detector is realised with a combination of a micro processor controller and a personal computer, allowing of a minimum integration time of $6.3 \mathrm{~ms}$.

The spectrometer is connected to the coupling optics via a $400 \mu \mathrm{m}$ NIR-fiber. This coupling optics consists of two convex lenses (L1: $200 \mathrm{~mm}, \mathrm{L2}: 25 \mathrm{~mm}$ ) [10], and allows of a free distance of $25 \mathrm{~cm}$ between the sample and L1. A 220V/150W halogen lamp illuminates a sample area of approximately $25 \mathrm{~cm}^{2}$. A brass plate of $5 \times 5 \mathrm{~cm}^{2}$ serves as reference to the spectrometer and back-illuminates transparent samples. Thus, the set-up enables to perform both, reflectance and transmission measurements. The plastic samples, that are cut out of plastic bodies taken from municipal waste, have a size of some $\mathrm{cm}^{2}$ and thicknesses from 0.5 to 
several $\mathrm{mm}$. The lenses collect scattered radiation from opaque samples as well as transmitted radiation from transparent samples; for translucent samples the detected radiation is a mixture of both contributions.

Before recording the spectra it is necessary to perform a reference measurement using the complete set-up without a sample in order to determine the reference intensity $\mathrm{I}_{\text {ref. }}$ Then the measured signal (S) is defined as $\mathrm{S}=\mathrm{I} / \mathrm{I}_{\mathrm{ref}}$, where $\mathrm{I}$ is the measured intensity.

The described set-up gives a SNR of at least 200:1 even in outer regions of the spectrum where the detector sensitivity decreases (at 824 or $1700 \mathrm{~nm}$ respectively).

\section{RESULTS AND DISCUSSION}

The plastic fraction in household garbage mainly consists of 5 plastic types, which are polyethylene (PE), polyethyleneterephthalate (PET), polypropylene (PP), polystyrene (PS) and polyvinylchloride (PVC). Figure 2 shows typical spectra of these five types of plastic. It can clearly be seen that there are differences between all of them in the spectral range covered by the used spectrometer.

A total number of 1009 spectra from 102 plastic samples (30 PE, 16 PET, 26 PP, 23 PS and 7 PVC) was obtained. For each sample about 10 different spectra were recorded, every spectrum for a different sample orientation. Black plastics were excluded because their NIRspectra do not reveal a characteristic structure of the material.

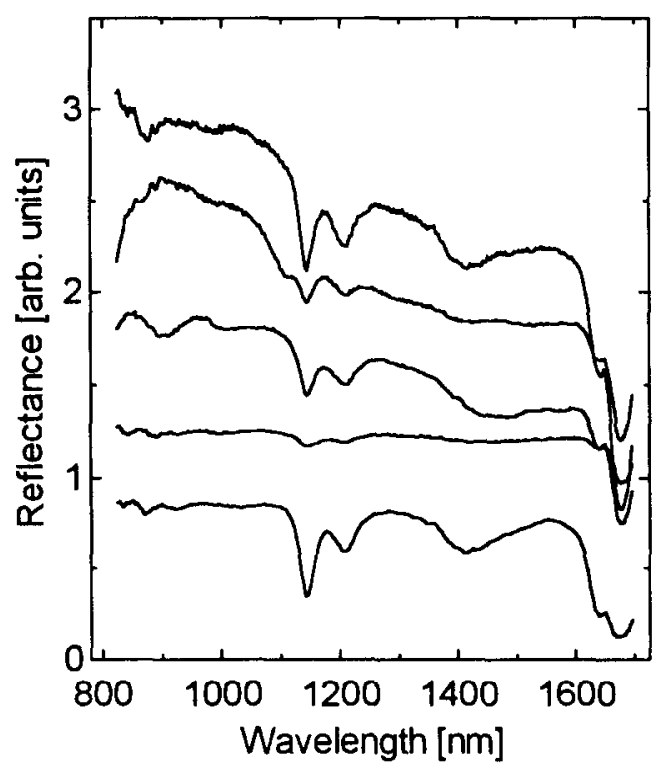

Figure 3. Spectra of different samples of polystyrene (PS)
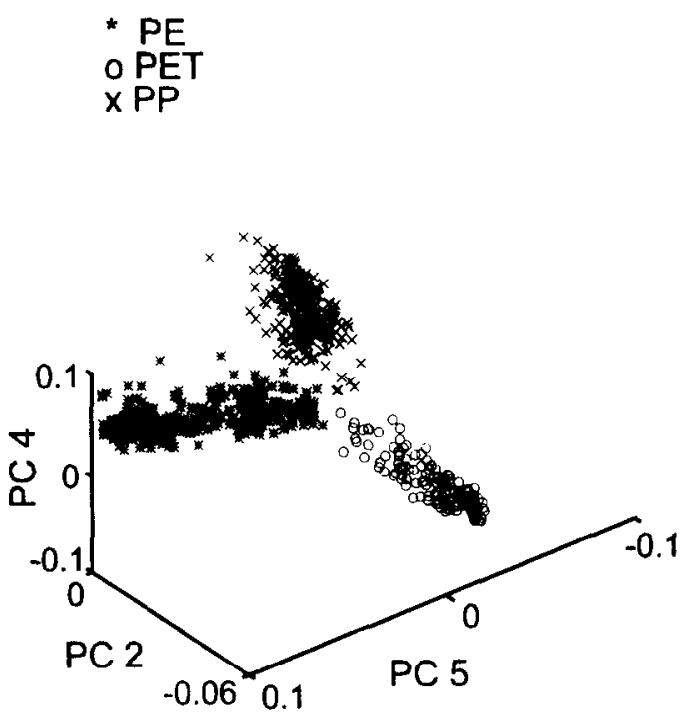

Figure 4. PCA cluster plot of preprocessed data from spectra of PE, PP or PET

Figure 3 shows spectra of 5 PS samples of different colour and transparency. One can see distinct variations in absolute absorption intensity as well as in the difference between maximum and minimum absorption value (dynamics) of the spectra within one class of plastics. 
This must be taken into account by the decision algorithm to be applied for polymer identification.

In figure 4 the results of a principal component analysis (PCA) of pre-processed (derivative, scaling) data, taken from spectra of PE, PP and PET, are illustrated. The areas corresponding to these three classes of plastics do not overlap, allowing of unambiguous identification. However, the separation of all 5 clusters is more complex and cannot be shown in a single $3 \mathrm{~d}-$ plot. It has been shown that the complete separation between all 5 classes can be achieved by neural network classifiers [9].

\section{CONCLUSIONS AND FUTURE DEVELOPMENTS}

It has been demonstrated that NIR spectra from 824 to $1700 \mathrm{~nm}$ of plastics with high signal to noise ratio can be obtained from a distance of up to $50 \mathrm{~cm}$ within 6.3 milliseconds. This integration time permits to record 158 spectra per second. Taking also into account the computation time of a neural network based decision algorithm as developed by Wienke et al. [9], 75 complete identification cycles can be performed per second. With this algorithm, a reliable distinction between the 5 major types of plastic contained in household garbage (PE, PET, PP, PS and PVC) was shown to be possible. We expect that this concept will provide the means for on-line identification of plastic garbage at usual speeds of industrial conveyor belts of up to $2 \mathrm{~m} / \mathrm{s}$. Further improvement of the identification system and it's application to real world garbage like entire bottles or plastic bodies with dirt or labels as well as it's adaptation to a moving belt are in progress. A detailed investigation of different pattern recognition techniques has been carried out [9] and will be further extended.

This work has been financed by the European Union, contract number EVWA-CT92-0001 and the 'Ministerium für Wissenschaft und Forschung, Nordrhein-Westfalen'.

\section{REFERENCES}

1. B.Willenberg, Kunststoffe, 84 (1994) 54.

2. H. Ritzmann, D. Schudel, Kunststoffe, 84 (1994) 582.

3. N. Eisenreich, H. Kull, E. Thinnes, Waste Management of Energetic Materials and Polymers (23rd Int. Annu. Conf. ICT, Karlsruhe) (1992) 59/1.

4. H. Lucht, U. Plauschin, H. Dürr, Umwelt, 23 No. 7/8 (1993) 443.

5. M. K. Alam, S. L. Stanton, Process Control and Quality, 4 (1993) 245

6. G. N. Foster, S. B. Row, R. G. Griskey, J. Appl. Polym. Sci., 8 (1964) 1357.

7. R. G. J. Miller, H. A. Willis, J. Appl. Chem., 6 (1956) 385.

8. M. J. Cohen, G. H. Olsen, SPIE Vol. 1946 Infrared Detectors and Instrumentation (1993) 436.

9. D. Wienke, W. van den Broek, R. Feldhoff, Th. Kantimm, Th. Huth-Fehre, L. Quick, W. Melssen, L. Buydens, K. Cammann - An Adaptive Resonance Theory Based Artificial Neural Network (Art-2a) for Rapid Sorting of Post Consumer Plastics by Remote Optical NIR Sensing with an InGaAs Diode Array Detector, in preparation

10. T. Huth-Fehre, T. Kantimm, R. Feldhoff, L. Quick, German Patent Application P 4423 770.7 (1994). 